\title{
Pandemic 2009 H1N1 virus infection associated with purpuric skin lesions: a case report
}

Rocco Urso ${ }^{1 *}$, Nazario Bevilacqua ${ }^{1}$, Marco Gentile², Daniele Biagioli and Francesco Nicola Lauria ${ }^{1}$

\begin{abstract}
Introduction: The influenza virus infection may be severe in non-immune people. Common complications of influenza virus include upper and lower respiratory tract infections, otitis media, myocarditis, acute respiratory distress syndrome and multi-organ failure. There have been cases of vasculitis following influenza vaccination, and rash and acute purpura may occur in certain viral infections. To the best of our knowledge, there are no reports concerning cases of systemic vasculitis associated with pandemic $2009\left(\mathrm{H}_{1} \mathrm{~N}_{1}\right)$ infection.

Case presentation: A 23-year-old Caucasian woman was hospitalized at the "L. Spallanzani" National Institute for Infectious Diseases in Rome, Italy. Clinical and radiological features including laboratory findings of this case are illustrated. Notably, the patient had fever, severe abdominal pain, hematuria, arthritis, and purpuric manifestations associated with a normal platelet count. Nasopharyngeal and rectal swabs revealed pandemic $2009\left(\mathrm{H}_{1} \mathrm{~N}_{1}\right)$ virus by reverse-transcriptase-polymerase-chain-reaction assay. Routine laboratory analyses showed elevated inflammatory parameters. The autoimmune panel tests were normal. Steroid therapy associated with oseltamivir achieved an evident and rapid improvement. On day seven the patient chose to leave the hospital against medical advice.

Conclusion: Complications related to influenza infection can be life threatening, particularly in immunocompromised patients. Henoch-Schönlein purpura triggered by the novel influenza virus infection could be an attractive pathogenetic hypothesis. We have discussed both the diagnosis and the challenge of therapy protocols. Steroid therapy is part of the management of severe vasculitis. Our case suggests that steroid therapy associated with antivirals can prevent the risk of further complications such as hemorrhage and multi-organ failure during severe vasculitis, without enhancing the virulence of the influenza virus. The possible role of pandemic 2009 $\left(\mathrm{H}_{1} \mathrm{~N}_{1}\right)$ in the pathogenesis of hemorrhagic manifestations should be further investigated.
\end{abstract}

\section{Introduction}

No cases of vasculitis were ever reported during the pandemic 2009 H1N1 (2009 H1N1) infection, thus its occurrence can be considered rare with undetermined etiology. Systemic vasculitis has been described as an extremely rare event during influenza infection or following the administration of vaccines [1-4]. In addition, influenza infection has seldom been associated with various autoimmune diseases [5]. Among viral respiratory diseases, adenovirus and enteroviruses were previously identified in patients with the onset of rash and cutaneous vasculitis [2,6]. Pandemic 2009 H1N1 had a broad clinical spectrum. The majority of 2009 H1N1 infections

\footnotetext{
* Correspondence: rocco.urso@inmi.it

'Infectious Respiratory Diseases Unit, Clinic Department, National Institute for Infectious Diseases "L. Spallanzani" (INMI), Rome, Italy

Full list of author information is available at the end of the article
}

are mild and self limiting; however, since the virus can cause serious respiratory disorders in young people and lead to severe complications in non-immune people, early treatment is recommended [7]. In the spring of 2009, the 2009 H1N1 virus spread rapidly throughout the world. The authors describe a case of systemic vasculitis that occurred in an Australian woman suffering from 2009 H1N1 infection. She was hospitalized at the "L. Spallanzani" National Institute for Infectious Diseases (INMI).

\section{Case presentation}

A 23-year-old Caucasian woman spending her holidays in Italy was admitted to INMI in Rome. The otherwise healthy patient presented with a two-day history of fever, severe abdominal pain, diarrhoea with bloody stools and haemorrhagic skin lesions.

\section{() Biomed Central}


Two weeks before, she had rhinorrhoea, a sore throat and a cough. She had received the first dose of human papillomavirus vaccination one month prior to her admittance. On examination, the patient looked pale, tired and feverish with a body temperature of $38.8^{\circ} \mathrm{C}$. She manifested an extension of haemorrhagic purpura all over her limbs (Figure 1) and complained of severe abdominal pain. Nasopharyngeal and rectal swabs evidenced a 2009 H1N1 virus by reverse-transcriptase polymerase chain reaction (RT-PCR) assay. Her blood count showed mild anaemia (haemoglobin, $11.70 \mathrm{~g} / \mathrm{dl}$ ), neutrophilia (white blood cells $15.700 / \mathrm{mmc}$ : neutrophils $13.400 / \mathrm{mmc}$ (85.1\%), lymphocytes $1.200 / \mathrm{mmc}(7,7 \%)$, monocytes $800 / \mathrm{mmc})$. C-reactive protein $(2.70 \mathrm{mg} / \mathrm{dl})$ and Dimer test values $(4.788 \mathrm{ng} / \mathrm{dl})$ were elevated. Platelet count, clotting tests and ESR were normal. A urine examination showed proteinuria, red cells, leukocytes, hyaline and erythrocyte casts. Specific laboratory tests were performed ruling out immunological and autoimmune disorders, including anti-nuclear antibody profile (autoantibodies against RNP/Sm, SS-A, Ro-52, SS-B,
Scl-70, Jo-1, centromere B, dsDNA, histones, anti-mitochondrial M2 antibody), anti-smooth muscle, anti-liverkidney microsomal antibodies, anti-platelets, anti-gastric parietal cell, anti-cardiolipin IgG and IgM, anti-neutrophilic cytoplasmic antibodies (ANCA), lupus anticoagulant, rheumatoid factor (RF), and Coombs test. Serological tests ruled out acute bacterial or viral infections including Brucella species, Salmonella species, Rickettsia conori, Coxiella burneti or viruses such as dengue, cytomegalovirus, Epstein-Barr virus, herpes simplex, HIV, coxsackievirus, echovirus and adenovirus. Bacterial nasopharyngeal, blood and urine cultures were sterile. Chest and abdomen X-rays were normal. A computed tomography (CT)-scan of her abdomen and pelvis without contrast revealed only a small effusion in the pouch of Douglas. Oseltamivir (75 mg twice a day, orally) and ciprofloxacin (400 mg twice a day, intravenously) were administered. A short course of methylprednisolone was administered intravenously, starting at $40 \mathrm{mg}$ on July 15 and then reduced by $10 \mathrm{mg}$ daily until it was withdrawn on July 20 . Her clinical condition

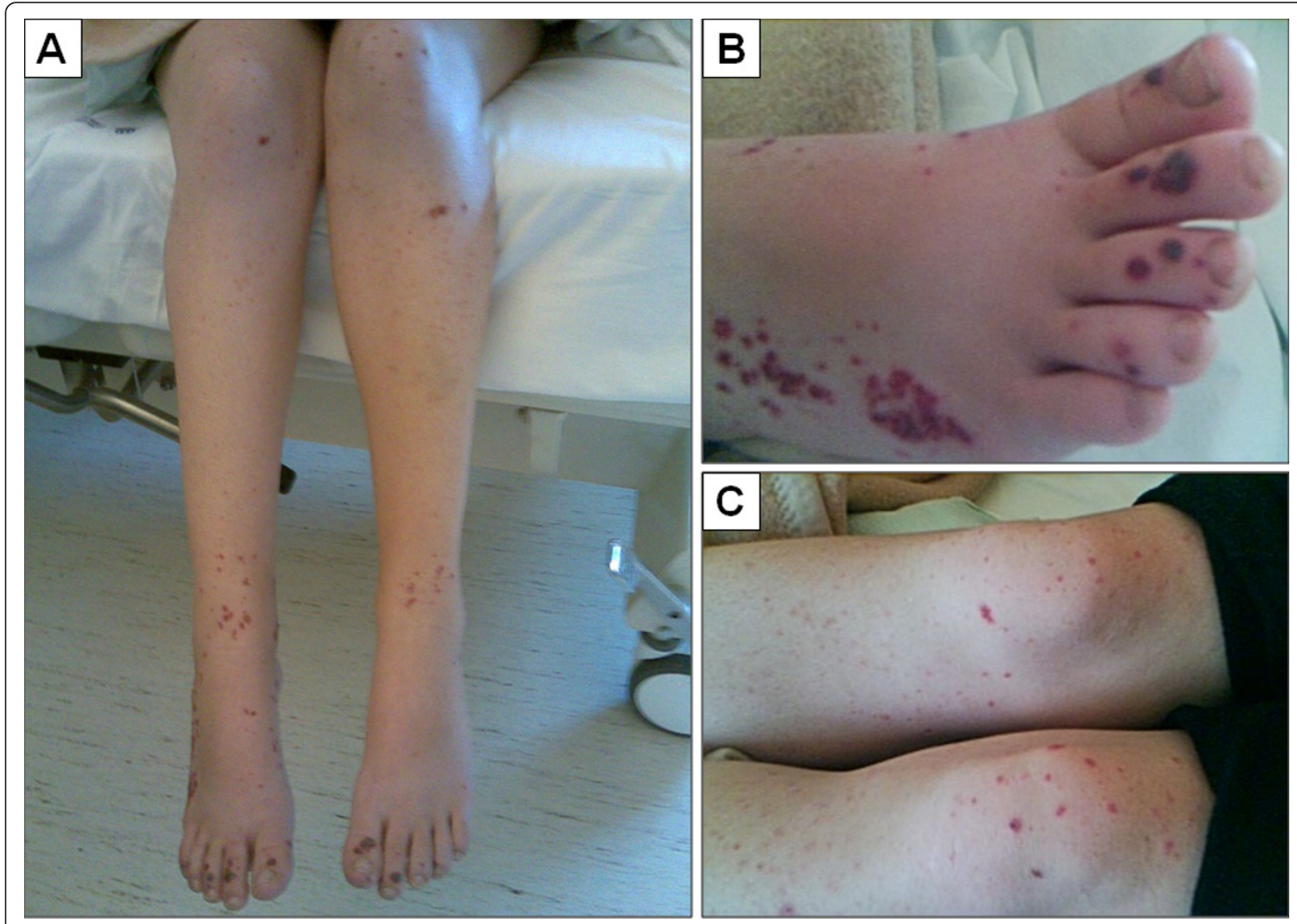

Figure 1 Overview of the features of purpura before treatment. A, C: show an overview of the features of purpura before treatment. B: details the hemorrhagic-necrotic skin lesions on the feet, associated with petechial lesions on the lateral part of the right foot. 
improved during hospitalization. By Day three the leukocytosis decreased (white blood cells $10.100 / \mathrm{mmc}$, neutrophils $5.600 / \mathrm{mmc}$, lymphocytes $3600 / \mathrm{mmc}$ ). The fever disappeared in three days, her abdominal pain was resolved and there was a marked improvement in the vasculitic lesions. On Day seven she chose to leave the hospital against medical advice.

\section{Discussion}

The rash that can occur during influenza virus infection usually has a macular or maculopapular aspect and can be confused with measles. Hope-Simpson and Higgins [8] found that approximately $8 \%$ of influenza B infections and $2 \%$ of influenza A virus infections are associated with rash. In addition, Silva and others [1] described a case of a child with a febrile petechial rash associated with influenza A virus infection.

Although cases of vasculitis have occurred following influenza vaccination [3,4], to our knowledge there are no reports concerning cases of systemic vasculitis associated with the $2009 \mathrm{H} 1 \mathrm{~N} 1$ infection. An immune thrombocytopenic purpura following human papillomavirus vaccination has been reported [9]. Other vasculitic conditions and sepsis were excluded.

The etiology of Henoch-Schönlein purpura (HSP), which is a systemic vasculitis described mainly in childhood $(0.013$ to $0.02 \%)$, actually remains unknown [10]. In our case, the skin and abdominal haemorrhagic manifestations associated with a normal platelet count could be related to both the 2009 H1N1 infection and HSP. Streptococcal, Mycoplasma species, adenovirus, parvovirus and coxsackievirus infections have been reported to precede HSP [10]. In our case, an upper respiratory tract infection occurred first. The main diagnostic hallmarks of HSP include clinical and histological features. In this case the clinical features of HSP such as fever, purpuric rash, bloody stools, hematuria, abdominal pain, joints and kidney involvement, and normal platelet count were present. We did not perform a biopsy of the skin lesions because of the voluntary discharge of the patient.

Vasculitis may result from the interaction between influenza virus antigen A H1N1 and the host's immune response. It has been hypothesized as being a pathogenetic immune-mediated mechanism induced by infections $[10,11]$. Deregulation of cytokine biosynthesis has been considered the fundamental pathogenetic mechanism related to the severity and progression of 2009 H1N1 influenza [12]. Particularly, the secretion of Th1 and Th17 cytokines was reported as an early host response in severe $2009 \mathrm{H} 1 \mathrm{~N} 1$ infection [13] and it is well known that these cytokines are also involved in inflammatory and autoimmune responses [14]. The proinflammatory immune response may facilitate the occurrence of vasculitis during a severe pandemic infection.
The spectrum of treatment against vasculitis during influenza infection has been broadly discussed. Steroid therapy was not absolutely contraindicated during 2009 H1N1 infection as reported in the scientific literature. Quispe-Laime and others reported that in patients with H1N1 influenza affected by severe respiratory complications, treatment with a prolonged, moderate dose of corticosteroids was associated with significant improvement in lung injury, multiple organ function scores and low hospital mortality [15]. Carter MJ et al. argued that steroids can have an additional role to antiviral therapy in the treatment of severe cases of H5N1 avian flu [13]. In our case, on the basis of a high clinical suspicion of severe vasculitis syndrome and considering the rapid impairment of the clinical conditions, a short-course of steroid therapy was added to the antiviral drugs. Despite the steroid therapy, by Day 6 the nasopharyngeal swab did not show evidence of the presence of 2009 H1N1 virus by RT-PCR. Randomized clinical trials are needed to determine whether additional steroid treatment could be beneficial to dominate spreading of the cytokines, which occurs in severe pandemic H1N1 influenza.

\section{Conclusion}

Systemic vasculitis associated with 2009 H1N1 infection is extremely rare. In this case, the role of the 2009 H1N1 influenza virus in the pathogenesis of hemorrhagic manifestations should be considered. The lack of a histopathological study of the skin lesions may limit our conclusions; however, the macroscopic characteristics of the purpura suggest a vasculitic process. Overall, the clinical, immunological and radiological features are consistent with the clinical diagnosis of HSP. In our case, a short course of steroid therapy in addition to antivirals did not delay the clearance of 2009 H1N1 virus and the combined treatment contributed to improving a complicated case of vasculitis.

\section{Consent}

Written informed consent was obtained from the patient for the publication of this case report and accompanying images. A copy of the written consent is available for review by the Editor-in-Chief of this journal.

\section{Abbreviations \\ 2009 H1N1: pandemic 2009 H1N1; HSP: Henoch-Schönlein purpura; INMI: "L. Spallanzani" National Institute for Infectious Diseases.}

\section{Acknowledgements}

We thank Mr. Nicola De Marco for his contribution of the images and we are grateful to Mr. Pierluca Piselli for the graphics support. There was no financial support for this study.

\section{Author details}

${ }^{1}$ Infectious Respiratory Diseases Unit, Clinic Department, National Institute for Infectious Diseases "L. Spallanzani" (INMI), Rome, Italy. ${ }^{2}$ Third Infectious 


\section{Authors' contributions}

$\mathrm{RU}$ and NB analyzed and interpreted the patient data regarding the

infectious issues and were the major contributors in writing the manuscript.

All authors read and approved the final manuscript.

\section{Competing interests}

The authors declare that they have no competing interests.

Received: 7 May 2010 Accepted: 1 April 2011 Published: 1 April 2011

\section{References}

1. Silva ME, Cherry JD, Wilton RJ, Ghafouri NM, Bruckner DA, Miller MJ: Acute fever and petechial rash associated with influenza A virus infection. Clin Infect Dis 1999, 29:453-454.

2. Pagnoux C, Cohen P, Guillevin L: Vasculitides secondary to infections. Clin Exp Rheumatol 2006, 24:S71-81.

3. Patel U, Bradley JR, Hamilton DV: Henoch-Schönlein purpura after influenza vaccination. Br Med J (Clin Res Ed) 1988, 296:1800.

4. Tishler M, Levi O, Amit-Vazina M: Immune thrombocytopenic purpura following influenza vaccination. Isr Med Assoc J 2006, 8:322-323.

5. Vilalta Castel E, Guerra Vales JM, González Gamarra A, Abarca Costalago M, Alonso Navas F, López Pascual JJ: Hemolytic anemia caused by cryoagglutinins associated with an influenza A virus infection. Rev Clin Esp 1986, 179:22-25.

6. Costa MM, Lisboa M, Romeu JC, Caldeira J, De Queiroz V: HenochSchőnlein purpura associated with coxsackie - virus B1 infection. Clin Rheumatol 1995, 14:488-490.

7. Redelman-Sidi G, Sepkowitz KA, Huang CK, Park S, Stiles J, Eagan J, Perlin DS, Pamer EG, Kamboj M: 2009 H1N1 influenza infection in cancer patients and hematopoietic stem cell transplant recipients. J Infect 2010, 60:257-263.

8. Hope-Simpson RE, Higgins PG: A respiratory virus study in Great Britain: review and evaluation. Prog Med Virol 1969, 11:354-407.

9. Pugnet $\mathrm{G}$, Isebaert L, Bagheri H, Montastruc JL, Laurent G: Immune thrombocytopenic purpura following human papilloma virus vaccination. Vaccine 2009, 27:3690.

10. Tizard EJ: Henoch- Schönlein purpura. Arch Dis Child 1999, 80:380-383.

11. Yang YH, Chuang YH, Wang LC, Huang HY, Gershwin ME, Chiang BL: The immunobiology of Henoch-Schőnlein purpura. Autoimmunity Reviews 2008, 7:179-184.

12. Woo PC, Tung ET, Chan KH, Lau CC, Lau SK, Yuen KY: Cytokine profiles induced by the novel swine-origin influenza $A / H 1 N 1$ virus: implications for treatment strategies. J Infect Dis 2010, 201:346-353.

13. Carter MJ: A rationale for using steroids in the treatment of severe cases of H5N1 avian influenza. J Med Microbiol 2007, 56:875-883, (Review).

14. Bermejo-Martin JF, Ortiz de Lejarazu R, Pumarola T, Rello J, Almansa R, Ramírez P, Martin-Loeches I, Varillas D, Gallegos MC, Serón C, Micheloud D, Gomez JM, Tenorio-Abreu A, Ramos MJ, Molina ML, Huidobro S, Sanchez E, Gordón M, Fernández V, Del Castillo A, Marcos MA, Villanueva B, López CJ, Rodríguez-Domínguez M, Galan JC, Cantón R, Lietor A, Rojo S, Eiros JM, Hinojosa C, Gonzalez l, et al: Th1 and Th17 hypercytokinemia as early host response signature in severe pandemic influenza. Crit Care 2009, 13: R201.

15. Quispe-Laime AM, Bracco JD, Barberio PA, Campagne CG, Rolfo VE, Umberger R, Meduri GU: H1N1 influenza A virus-associated acute lung injury: response to combination oseltamivir and prolonged corticosteroid treatment. Intensive Care Med 2010, 36:33-41.

\section{doi:10.1186/1752-1947-5-132}

Cite this article as: Urso et al:: Pandemic 2009 H1N1 virus infection associated with purpuric skin lesions: a case report. Journal of Medical Case Reports 2011 5:132. 